\title{
Unusual disseminated Talaromyces marneffei infection mimicking lymphoma in a non-immunosuppressed patient in East China: a case report and review of the literature
}

Donghe Chen ${ }^{1}$, Chengdong Chang ${ }^{2}$, Ming Chen ${ }^{3}$, Yafei Zhang ${ }^{1}$, Xin Zhao ${ }^{1}$, Tingting Zhang ${ }^{1}$, Zhen Wang ${ }^{1}$, Jing Yan ${ }^{1}$, Huanyan Zhu', Lin Zheng ${ }^{4}$ and Kui Zhao ${ }^{1 *}$

\begin{abstract}
Background: Talaromyces marneffei infection is an important opportunistic infection associated with acquired immune deficiency syndrome (AIDS). However, it is unusual in patients with non-AIDS and other non-immunosuppressed conditions. We report a case of delayed diagnosis of disseminated T. marneffei infection in non-AIDS, non-immunosuppressive and nonendemic conditions.

Case presentation: We describe a previously healthy 24-year-old man who complained of a 3-month history of intermittent diarrhea and a recent week of uncontrollable high fever. The HIV antibody test was negative. Enhanced abdominal computed tomography (CT) and integrated ${ }^{18} \mathrm{~F}$-2-deoxy-2-fluoro-D-glucose position emission tomography/computed tomography (FDG PET/CT) both suspected malignant lymphoma. However, a large number of yeast-like cells were found in macrophages in cervical lymph node samples by hematoxylin and eosin stain and silver hexamine stain. Subsequent blood culture suggested T. marneffei infection. Metagenomic Next Generation Sequencing (mNGS) results suggested T. marneffei as the dominant pathogen. Unfortunately, the patient continued to develop acute liver failure and died due to adverse events associated with amphotericin B.
\end{abstract}

Conclusions: Early diagnosis in HIV-negative patients who are otherwise not immunosuppressed and endemic poses a serious challenge. T. marneffei infection is an FDG-avid nonmalignant condition that may lead to false-positive FDG PET/CT scans. Nevertheless, integrated FDG PET/CT is necessary in patients with fever of unknown origin in the early period to perform earlier biopsy for histopathology and culture in highly avid sites and to avoid delays in diagnosis and treatment.

Keywords: Talaromyces marneffei, Non-AIDS patient, Non-immunosuppressed patient, FDG PET/CT, Delay in diagnosis

\footnotetext{
* Correspondence: zhaokui0905@zju.edu.cn

'PET Center, The First Affiliated Hospital, Zhejiang University School of Medicine, Hangzhou, Zhejiang 310003, P.R. China

Full list of author information is available at the end of the article
}

(c) The Author(s). 2020 Open Access This article is licensed under a Creative Commons Attribution 4.0 International License, which permits use, sharing, adaptation, distribution and reproduction in any medium or format, as long as you give appropriate credit to the original author(s) and the source, provide a link to the Creative Commons licence, and indicate if changes were made. The images or other third party material in this article are included in the article's Creative Commons licence, unless indicated otherwise in a credit line to the material. If material is not included in the article's Creative Commons licence and your intended use is not permitted by statutory regulation or exceeds the permitted use, you will need to obtain permission directly from the copyright holder. To view a copy of this licence, visit http://creativecommons.org/licenses/by/4.0/ The Creative Commons Public Domain Dedication waiver (http://creativecommons.org/publicdomain/zero/1.0/) applies to the data made available in this article, unless otherwise stated in a credit line to the data. 


\section{Background}

Talaromyces marneffei infection is a disseminated and progressive infection and is recognized as an important opportunistic fungal infection in patients who live in or travel to areas of endemicity, including Southeast Asia, Thailand, Hong Kong, and the southern part of China [1-5]. The reservoirs of T. marneffei in nature include bamboo rats [6] and the soil [2], which lead to human infections, especially in the moist environment of the concurrent rainy season $[1,2]$.

Infection has been widely suggested to be an indicator disease for differential diagnosis in HIV-infected patients
$[4,5,7]$. In recent years, it has been reported that an increasing number and proportion of cases in non-HIVinfected patients who had some form of immunodeficiency (hypogammaglobulinemia, CD4 lymphopenia) or underlying diseases with immunocompromised condition, including hematologic malignancies or autoimmune diseases, were receiving treatments that could impair their cellular immunity, such as antineoplastic or corticosteroid agents $[8,9]$. However, some researchers have recommended that $T$. marneffei infection also occur in persons with normal immunity $[4,8,10-12]$. Here, we report an unusual case of disseminated $T$.

Table 1 Clinical laboratory results

\begin{tabular}{|c|c|c|c|}
\hline Measure & Reference Range & Patient On Admission & Patient With Acute Liver Failure \\
\hline White-cell count (10E9/L) & $4.0-10.0$ & $2.9 \downarrow$ & $20.3 \uparrow \uparrow$ \\
\hline Absolute neutrophil count (10E9/L) & $2.0-7.0$ & $0.8 \downarrow$ & $17.4 \uparrow \uparrow$ \\
\hline Absolute lymphocyte count (10E9/L) & $0.8-4.0$ & 1.65 & 1.27 \\
\hline Red-cell count (10E12/L) & $4.09-5.74$ & 5.14 & $3.68 \downarrow$ \\
\hline Hemoglobin (g/dl) & $131-172$ & $115 \downarrow$ & $102 \downarrow$ \\
\hline Platelet count (10E9/L) & $83-303$ & 281 & $32 \downarrow$ \\
\hline $\mathrm{CRP}^{\mathrm{a}}(\mathrm{mg} / \mathrm{L})$ & $0.00-8.00$ & $96.48 \uparrow \uparrow$ & $21.2 \uparrow$ \\
\hline $\operatorname{ESR}^{\mathrm{a}}(\mathrm{mm} / \mathrm{h})$ & $0-15$ & $43 \uparrow$ & NA \\
\hline Sodium (mmol/L) & $136-145$ & $128 \downarrow$ & 150 \\
\hline Potassium (mmol/L) & $3.50-5.20$ & $2.81 \downarrow$ & $2.64 \downarrow$ \\
\hline Chloride (mmol/L) & $96-108$ & $89 \downarrow$ & 109 \\
\hline Calcium (mmol/L) & $2.03-2.54$ & $1.88 \downarrow$ & $1.78 \downarrow$ \\
\hline Magnesium (mmol/L) & $0.87-1.45$ & 0.75 & 1.86 \\
\hline Inorganic phosphorus (mmol/L) & $0.87-1.45$ & 0.95 & 0.98 \\
\hline Blood urea nitrogen (mmol/L) & $2.90-8.20$ & $2.51 \downarrow$ & 5.10 \\
\hline Creatinine $(\mu \mathrm{mol} / \mathrm{L})$ & 59-104 & $49 \downarrow$ & 54 \\
\hline Total protein $(\mathrm{g} / \mathrm{L})$ & $64.0-83.0$ & $55.3 \downarrow$ & NA \\
\hline Albumin $(g / L)$ & $35.0-55.0$ & $31.7 \downarrow$ & NA \\
\hline Fasting blood-glucose (mmol/L) & $3.90-6.10$ & 5.99 & NA \\
\hline Adenylic deaminase (U/L) & $0-18$ & $29 \uparrow$ & NA \\
\hline Alanine aminotransferase (U/L) & $5-40$ & 22 & $2161 \uparrow \uparrow$ \\
\hline Aspertate Aminotransferase (U/L) & $3-50$ & 20 & $1943 \uparrow \uparrow$ \\
\hline Lactate dehydrogenase (U/L) & $109-245$ & NA & $919 \uparrow \uparrow$ \\
\hline Total bilirubin ( $\mu \mathrm{mol} / \mathrm{L})$ & $0-21$ & 11 & $58 \uparrow$ \\
\hline Blood lactate (mmol/L) & $0.5-1.6$ & NA & $27 \uparrow \uparrow$ \\
\hline $\mathrm{PCO}_{2}{ }^{\mathrm{a}}(\mathrm{mmHg})$ & $35-45$ & NA & $9.4 \downarrow \downarrow$ \\
\hline Bicarbonate concentration (mmol/L) & $22-27$ & NA & $6.5 \downarrow \downarrow$ \\
\hline Blood ammonia ( $\mu \mathrm{mol} / \mathrm{L})$ & $10-47$ & NA & $500 \uparrow \uparrow$ \\
\hline Activated coagulation time of whole blood(s) & $22.0-36.0$ & 30.4 & $72.2 \uparrow \uparrow$ \\
\hline Thrombin time(s) & $14.5-21.5$ & 16.7 & $23.6 \uparrow$ \\
\hline Prothrombin time(s) & $10.0-13.5$ & $14.6 \uparrow$ & $>150 \uparrow \uparrow$ \\
\hline D-dimer ( $\mu \mathrm{g} / \mathrm{L}$ FEU) & $0-700$ & $2093 \uparrow$ & $5577 \uparrow \uparrow$ \\
\hline
\end{tabular}

${ }^{a} C R P$ C-reactive protein, $E S R$ Erythrocyte sedimentation rate, $P C O_{2}$ Partial pressure of carbon dioxide. $\downarrow$ : Mild to moderate reduction. $\uparrow:$ Mild to moderate elevation. $\downarrow \downarrow$ : Severe reduction. $\uparrow \uparrow$ : Severe elevation. NA: No measurement 
marneffei infection in non-HIV, non-immunosuppressed and non-endemic conditions.

\section{Case presentation}

A previously healthy 24-year-old male college student presented to our hospital with a 3-month history of intermittent diarrhea and a recent week of uncontrollable high fever, for which he was prescribed intravenous cefodizime sodium and emeticin against infection without any improvement in local hospitals. He denied a history of intravenous drug use, homosexual activity, or transfusion of blood products. He had no known history of any definitive systemic disease. His parents were healthy, and he had a brother who died of aplastic anemia at the age of six.

On admission, the vital signs revealed a body temperature of $40^{\circ} \mathrm{C}$, blood pressure of $99 / 56 \mathrm{mmHg}$, pulse of 89 beats per minute, and respiratory rate of 20 breaths per minute. The physical examination revealed a very gaunt and fatigued patient with a scaphoid abdomen. The liver, spleen and superficial lymph nodes were not palpable.

Initial laboratory results at the hospital reflected leukopenia, hypohemoglobin, hypokalemia, hyponatremia and elevated levels of creatine kinase (CRP) and erythrocyte sedimentation rate (ESR). In addition, there were alterations in hepatic and immunologic function measures: the level of adenylic deaminase was elevated, and serum levels of immunoglobulin G (IgG), total protein, and albumin were all decreased (Table 1). The HIV antibody test was negative, and cytomegalovirus (CMV) detection was positive. Given the patient's recurrent fevers, blood cultures and marrow aspirate were obtained on hospitalization.

After admission, the patient received antibacterial treatment and supportive care, including replenishing electrolytes, fluid and human serum albumin.

An enhanced abdominal CT taken the next day showed significant lymph node enlargement and multiple thickening of the intestinal wall, and malignant disease, especially lymphoma, was suspected (Fig. 1a, b, c). Integrated FDG $\mathrm{PET} / \mathrm{CT}$ was performed for characterization and staging of this suspicious disease, revealing hypermetabolic cervical, retroperitoneal and mesenteric lymph node (the maximum standardized uptake value $\left(\mathrm{SUV}_{\max }\right)$ 8.7) associated with diffuse hypermetabolism in the thickened intestinal wall (SUV $V_{\max }$ 11.2) (Fig. 2).

Clinical, biological and radiological presentations were suggestive of a malignant lymphoma. Subsequently, the patient received surgical biopsy in the cervical hypermetabolic lymph node. Colonoscopy indicated multiple ulcerations of the colon with local biopsy (Fig. 1d).

On days 10 through 14 of hospitalization, the patient's vital signs remained largely stable. Intestinal biopsy suggests chronic moderate-reactive inflammation of the colon mucosa with ulceration, and marrow aspirate suggests marked granulocytic hyperplasia and does not show any lymphomatous or other malignant disease. Surgical biopsy showed that the basic structure of the lymph node still existed, with hyperplasia and coagulative necrosis in lymphoid tissue, but presented a number of yeast -like cells in macrophage by
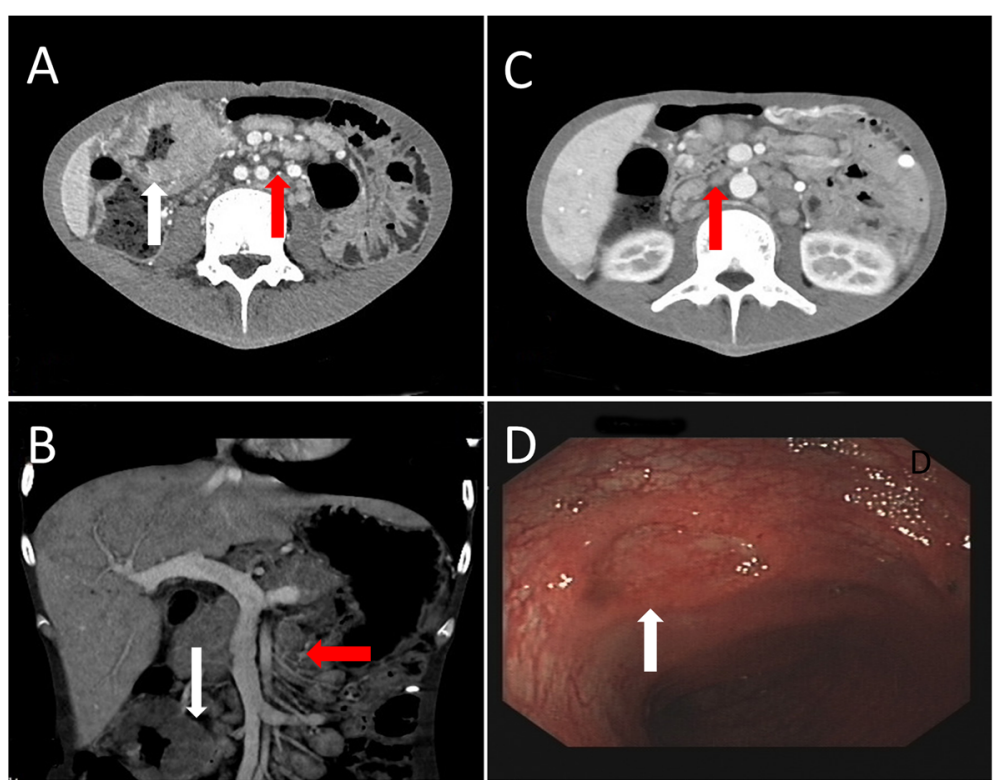

Fig. 1 Enhanced abdominal CT (a, b, c) showed significant lymph node enlargement (red arrows) and multiple thickening of the intestinal wall (white arrows). Colonoscopy indicated multiple ulcerations of the colon (d, white arrow) 

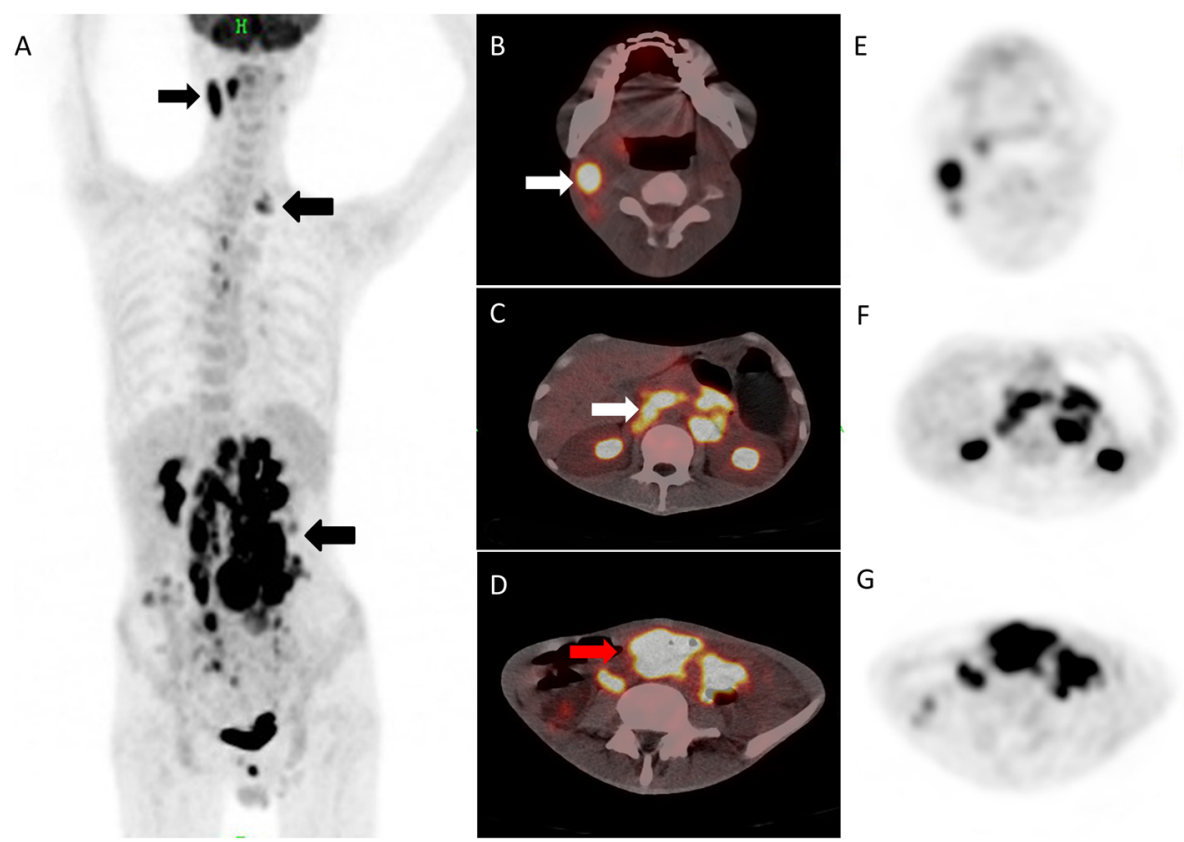

Fig. 2 The MIP of ${ }^{18}$ F-FDG PET/CT (a) revealed multiple hypermetabolic lesions in the whole body (black arrows). Axial slices showed cervical, retroperitoneal and mesenteric lymph node enlargement $\left(S U V_{\max }=11.1\right.$, white arrows) and diffusely thickened intestinal wall $\left(S U V_{\max }=10.3\right.$, red arrow) on PET/CT fusion (b-d) and PET (e-g)

hematoxylin and eosin staining (Fig. 3a) and hexamine staining (Fig. 3b) at a magnification of 1000. At the same time, blood culture and surprisingly suggested T. marneffei infection (Fig. 3c, d). The mNGS results suggested T. marneffe $i$ as the dominant pathogen (Fig. 4a, b).

The patient began intravenous amphotericin B (0.6 $\mathrm{mg} / \mathrm{kg} /$ day) and voriconazole according to current infectious disease guidelines. On hospital day 20 (after treatment with amphotericin B for 7 days), the patient suddenly presented with irritability and developed unconsciousness with gatism $2 \mathrm{~h}$ later. The vital signs were a body temperature of $37^{\circ} \mathrm{C}$, blood pressure of $123 / 71 \mathrm{mmHg}$, pulse of 132 beats per minute, respiratory rate of 29 breaths per minute, and oxygen saturation of $99 \%$, while the patient was breathing ambient air. The physical examination revealed coma with dilated pupils $(0.3 \mathrm{~cm})$. In addition, laboratory results showed that the levels of blood ammonia, white-cell count, lactate dehydrogenase (LDH), aspartate aminotransferase (AST), alanine aminotransferase (ALT), and prothrombin time (PT) were all significantly increased. Blood gas analysis revealed life-threatening elevation in lactic acid $(27 \mathrm{mmol} / \mathrm{L})$ and reduction in partial pressure of carbon dioxide $(9.4 \mathrm{mmHg}$ ) (Table $1)$. The patient continued to develop acute liver failure with hepatic encephalopathy and prothrombin time prolongation and died 1 week later.

\section{Discussion and conclusion}

T. marneffei infection has been considered to be an important opportunistic infection associated with AIDS. However, it is unusual in patients with non-AIDS and other non-immunosuppressed conditions. Chan JF et al. [4] reported that adult-onset immunodeficiency syndrome caused by anti-interferon-gamma (anti-IFN- $\gamma$ ) autoantibodies might help to explain many previous cases of $T$. marneffei infection in non-HIV patients who had no other comorbidities. We also believe our patient may have had an underlying immunosuppressive disorder that remained undefined even after an extensive investigation.

T. marneffei appears to be a primary pulmonary pathogen acquired by inhalation of spores, which may cause acute pulmonary disease, dissemination to the skin, reticuloendothelial system, bone marrow, gut and other organ systems by hematogenous spread [2, 3]. Phagocytic cells are the primary host defense against the fungus, resulting in granulomatous and suppurative reactions in immunocompetent patients and necrotizing reactions in those who are immunocompromised. However, in our case, since recurrent diarrhea was the only early symptom, we considered that the intestine might be the site of primary infection via ingestion of organism.

The severity and clinical manifestations of disease depend on the degree of host immunosuppression [2-4]. $T$. marneffei mostly causes mild and localized infections in 


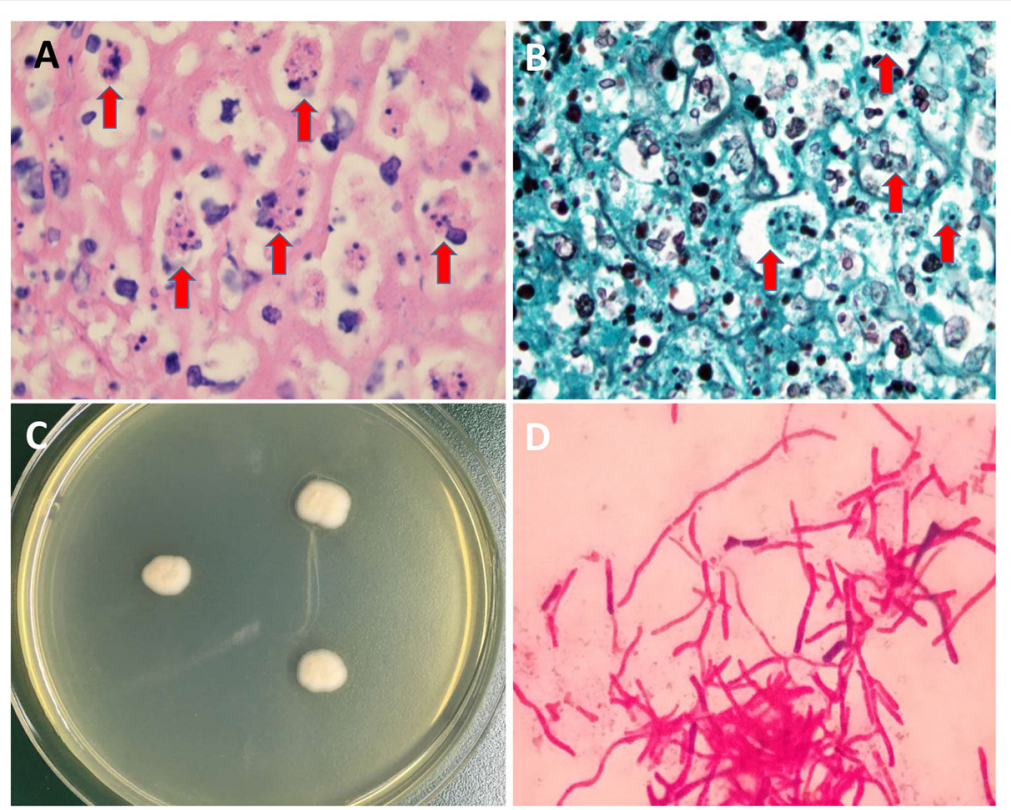

Fig. 3 Pathological examination showed numerous yeast-like cells in macrophages (red arrows) in cervical lymph node samples by hematoxylin and eosin stain (a, Magnification, $\times 1000$ ) and silver hexamine stain (b, Magnification, $\times 1000$ ). Blood culture (after 4 days incubation at $25^{\circ} \mathrm{C}$ ) surprisingly suggested T. marneffei infection (a). The mold was smeared for Gram staining from blood culture showing the red and rod-shaped hyphae (d, Magnification, $\times 1000$ )

patients with normal immunity, but it can cause severe disseminated infections with generalized lymphadenopathy and persistent fever in AIDS patients [13, 14]. However, many previous articles have reported that varying clinical manifestations of T. marneffei infection, including fever, lymphadenopathy, hepatosplenomegaly, malaise, weight loss, skin and soft tissue lesions, cough and dyspnea, commonly occurred among these non-HIV patients $[2,4]$. Because of nonspecific clinical manifestations, $T$. mameffei infection can be easily misdiagnosed as tuberculosis, histoplasmosis, cryptococcosis and lymphoma in patients with fever and generalized lymphadenopathy $[4,7]$. Chan JF et al. [4] reported that some patients who had osteoarticular involvement and abdominal symptoms such as abdominal pain and diarrhea mimicked Crohn's disease. In our patient, abdominal symptoms and fever due to mesenteric lymphadenopathy or colonitis were the major but nonspecific clinical symptoms, leading to incorrect suspicion of malignant lymphoma.

Laboratory manifestations of patients with $T$. marneffei infection are less described in previous literature. Only a few articles $[7,15]$ considered that anemia was the most consistent laboratory abnormality, especially severe anemia, which was much more severe than that usually encountered in anemia of chronic disease. Laboratory assessments of our patient on admission, consisting of complete blood count, blood chemistry, coagulation function, renal and liver function, and electrolytes, revealed obvious hypoleukemia, hypohemoglobinemia, hypoproteinemia and hypokalemia, possibly due to chronic recurrent diarrhea and malnutrition. In addition, significant increases in temperature, ESR and serum levels of CRP, relating to the magnitude of the inflammatory response, indicated severe fungal activity in his body. Therefore, low electrolyte levels, low hemoglobin and neutrophils can also be considered characteristic laboratory manifestations in non-AIDS patients with $T$. marneffei infection, which is helpful in making early and correct diagnoses.

The underlying diseases such as AIDS and other immunodeficiencies, as well as a history of travel to an endemic area, can help a physician make the appropriate or suspected diagnosis [3]. Microbiological culture from a variety of specimens, including blood, skin, bone marrow, lymph nodes, respiratory sources, liver, cerebrospinal fluid, urine, stool, kidney, pericardium, intestine or stomach, remains the gold standard for the diagnosis of $T$. marneffei infection [3, 7]. Additionally, the mortality rate is high, at approximately $75 \%$ in those who delay the diagnosis and administration of antifungal therapy regardless of whether HIV infection is involved [7, 16]. Consistent with previous reports $[4,7,9]$, the delayed diagnosis of our case was established through blood culture 3 months after the onset of symptoms due to the lack of clinical suspicion in the early stage. Hence, early diagnosis in HIV-negative patients who are otherwise not immunosuppressed and endemic still pose a serious challenge.

Integrated FDG PET/CT is a wide imaging technique used in the evaluation of diverse oncological indications. 
A

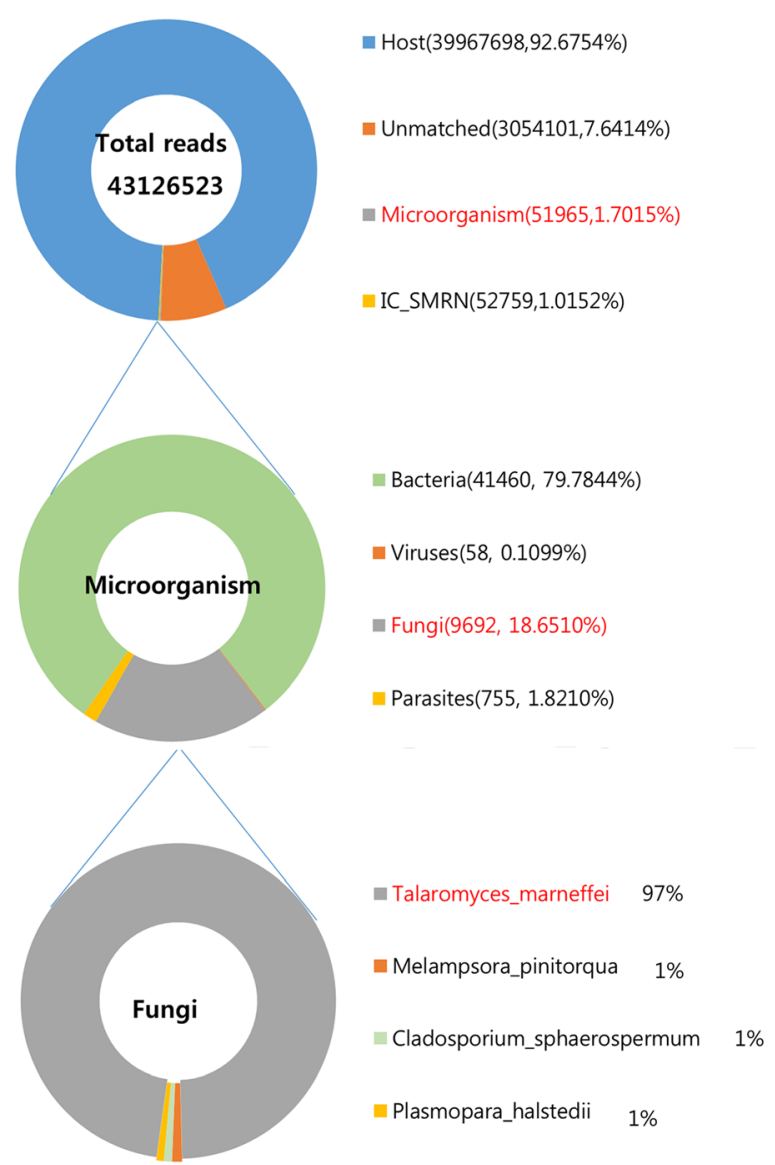

B

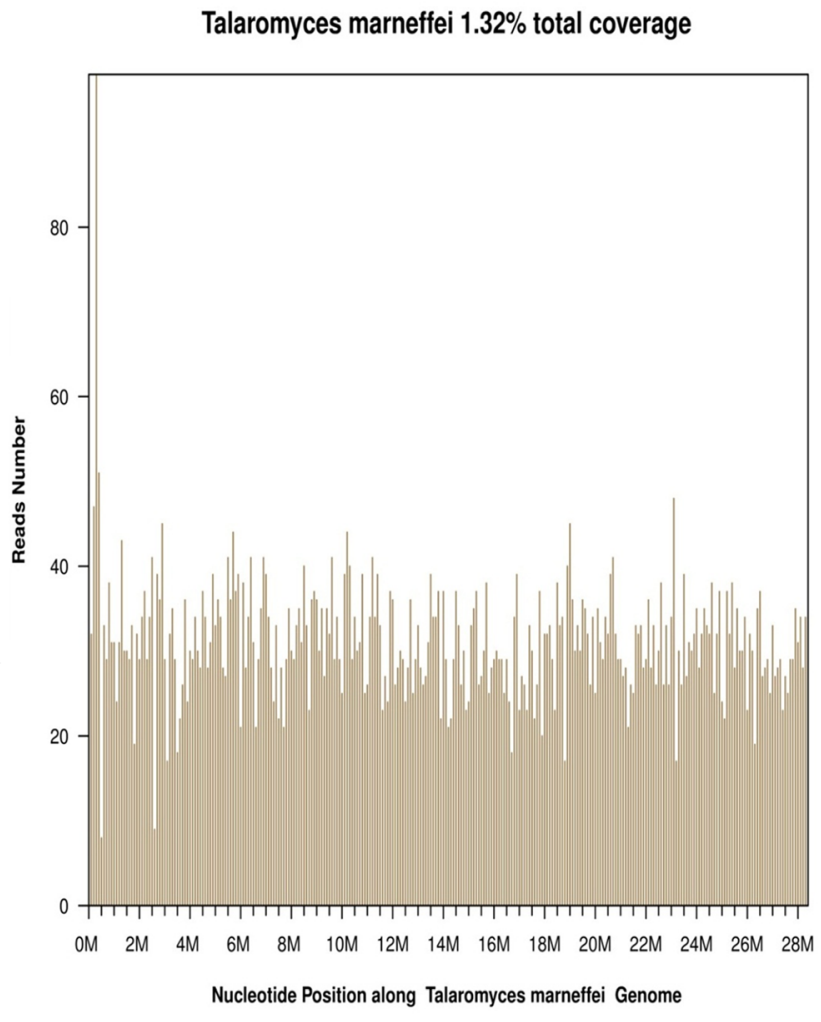

Fig. 4 The proportions of the identified sequencing suggested T.marneffei as the dominant pathogen. The number of mapped reads and percentage is given in the total 43,126,523 reads. The number of microbial reads was 51,965(1.7015\%), and the number of the fungal was 9692 (18.6510\%). T.marneffei had the highest relative abundance at 97\%, suggesting that it was the dominant pathogen (a). Sequencing of the isolated strain was conducted and a total coverage of $1.32 \%$ was obtained (b)

It is being increasingly recognized that FDG PET/CT has clinical applications beyond the remit of cancer imaging with addition value in fields of non-oncological conditions, including suspected infection and inflammation $[17,18]$. Early performing FDG PET/CT imaging has been shown to more accurately detect clinically occult neoplasm or sources of the fever and identify additional disease sites compared to conventional imaging, and guide appropriate treatment [19-21]. In addition, many previous studies $[14,16,22,23]$ revealed that generalized lymphadenopathy, hepatosplenomegaly and disseminated infection with multi-organ involvement were commonly observed in T. marneffei infections. In our patient, FDG PET/CT showed hypermetabolic generalized lymphadenopathy and intestinal lesions on week 2 of hospitalization (fever of unknown origin day 12). Although we performed cervical lymph node fine-needle aspiration and colonoscopy in time under PET/CT guidance, it still took 10 days to wait for pathology and 2 weeks for blood culture (on weeks 3 through 4 of hospitalization). There is a certain delay in the diagnosis because the patient and initial doctors do not attach importance to this serious disease. We believe that the delay in diagnosis could be avoided if patients undergo whole-body FDG PET/CT in the early period of fever or earlier onset. Although it may be difficult to differentiate infectious or inflammatory lesions from malignant tumors solely by the intensity of FDG uptake, we can perform early aspiration cytology of hypermetabolic lesions such as lymph nodes, liver and bone marrow commonly involved organs in T. marneffei infections, especially in HIV-negative hosts. The use of this method of diagnosis is also potentially beneficial to patients in whom lymphadenopathy or other occult lesions are confined to deep areas.

Amphotericin B is the preferred drug to treat T. marneffei infection, especially in severe cases [7]. However, the adverse effects of amphotericin B, such as severe anemia, electrolyte imbalances, nephrotoxicity, and hepatotoxicity, occur frequently and limit its clinical 
application [24]. Our patient developed progressive liver function impairment and eventually acute liver failure after 7 days of treatment with amphotericin B and voriconazole. The most likely reason may be the adverse events associated with amphotericin B in patients with severe systemic inflammatory response syndrome and malnutrition.

We reported a rare case of disseminated T. marneffei infection whose gut might be the site of primary infection via ingestion of organisms. Key aspects of this case included awareness of the potential infection in nonAIDS, non-immunosuppressed and non-endemic patients in China; recognition of fatal outcome due to the delay in diagnosis and treatment; and identification of the value of FDG PET/CT in infection and inflammation. From this report, low electrolyte levels, hemoglobin and neutrophils may be considered characteristic laboratory manifestations in severe infection by $T$. marneffei. In addition, it is clear that $T$. marneffei infection is an FDG-avid nonmalignant condition that may lead to false positives on FDG PET/CT scans. This case report also highlights the necessity of FDG PET in patients with fever of unknown in the early period to earlier perform biopsy for histopathology and culture in highly avid sites and to avoid delays in diagnosis and treatment.

\begin{abstract}
Abbreviations
AIDS: Acquired immune deficiency syndrome; CT: Computed tomography; FDG: ${ }^{18}$ F-2-deoxy-2-fluoro-D-glucose; PET/CT: Position emission tomography/ computed tomography; HIV: Human immunodeficiency virus; CRP: Creatine kinase; ESR: Erythrocyte sedimentation rate; IgG: Immunoglobulin G;

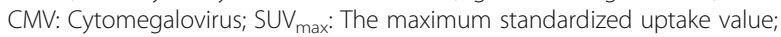
LDH: Lactate dehydrogenase; AST: Aspertate aminotransferase; ALT: Alanine aminotransferase; PT: Prothrombin time; Anti-IFN- $\gamma$ : Anti-interferon-gamma
\end{abstract}

\section{Acknowledgements}

We are thankful to the parents of patient for the support given in providing the data.

\section{Authors' contributions \\ DC: Study conception and design; drafting of manuscript; critical revision; review, analysis and interpretation of scientific literature. \\ CC: Management of the specimen samples of the patient; drafting of manuscript. MC: Analysis of mNGS data and production of sequencing coverage map. KZ, YZ, ZX, TZ, JY, ZW, HZ and LZ: Drafting of manuscript; review, analysis and interpretation of scientific literature. All authors have contributed to the manuscript and approved its final version.}

\section{Funding}

No outside funding was utilized for this project.

\section{Availability of data and materials}

The datasets used and/or analyzed during the current study are available from the corresponding author on reasonable request.

\section{Ethics approval and consent to participate}

The study was approved by the Ethics Committee of The first Affiliated Hospital, College of Medicine, Zhejiang University.

\section{Consent for publication}

Written informed consent was obtained from the patient's next of kin for publication of this case report and any accompanying images. A copy of the written consent is available for review by the Editor-in-Chief of this journal.

\section{Competing interests}

The authors declare that they have no competing interests.

\section{Author details}

${ }^{1}$ PET Center, The First Affiliated Hospital, Zhejiang University School of Medicine, Hangzhou, Zhejiang 310003, P.R. China. ${ }^{2}$ Department of Pathology, The First Affiliated Hospital, Zhejiang University School of Medicine, Hangzhou, Zhejiang 310003, P.R. China. ${ }^{3}$ BGI Genomics, Shenzhen, Guangdong 518083, P.R. China. ${ }^{4}$ State Key Laboratory for Diagnosis and Treatment of Infectious Diseases, Hangzhou, Zhejiang 310003, P.R. China.

Received: 15 March 2020 Accepted: 16 October 2020

Published online: 28 October 2020

\section{References}

1. Li HR, Xu NL, Lin M, Hu XL, Chen JH, Chen YS, Cai SX. Diffuse interstitial and multiple cavitary lung lesions due to Talaromyces marneffei infection in a non-HIV patient. New Microbes New Infections. 2015;8:14-6.

2. Vanittanakom N, Cooper CR Jr, Fisher MC, Sirisanthana T. Penicillium marneffei infection and recent advances in the epidemiology and molecular biology aspects. Clin Microbiol Rev. 2006;19(1):95-110.

3. Castro-Lainez MT, Sierra-Hoffman M, J LL-Z, Adams R, Howell A, HoffmanRoberts H, Fader R, Arroliga AC, Jinadatha C: Talaromyces marneffei infection in a non-HIV non-endemic population. IDCases 2018, 12:21-24.

4. Chan JF, Lau SK, Yuen KY, Woo PC. Talaromyces (Penicillium) marneffei infection in non-HIV-infected patients. Emerging Microbes Infections. 2016;5:e19.

5. Kawila R, Chaiwarith R, Supparatpinyo K. Clinical and laboratory characteristics of penicilliosis marneffei among patients with and without HIV infection in northern Thailand: a retrospective study. BMC Infect Dis. 2013:13:464.

6. Ajello L, Padhye AA, Sukroongreung S, Nilakul $\mathrm{CH}$, Tantimavanic $\mathrm{S}$. Occurrence of Penicillium marneffei infections among wild bamboo rats in Thailand. Mycopathologia. 1995;131(1):1-8.

7. Chen J, Zhang R, Shen Y, Liu L, Qi T, Wang Z, Song W, Tang Y, Lu H. Clinical characteristics and prognosis of Penicilliosis among human immunodeficiency virus-infected patients in eastern China. Am J Tropical Med Hygiene. 2017;96(6):1350-4.

8. Lee PP, Lao-Araya M, Yang J, Chan KW, Ma H, Pei LC, Kui L, Mao H, Yang W, Zhao X, et al. Application of flow Cytometry in the diagnostics pipeline of primary Immunodeficiencies underlying disseminated Talaromyces marneffei infection in HIV-negative children. Front Immunol. 2019;10:2189.

9. Wong SSY, Wong KH, Hui WT, Lee SS, Lo JYC, Cao L, Yuen KY. Differences in clinical and laboratory diagnostic characteristics of Penicilliosis Marneffei in human immunodeficiency virus (HIV)- and non-HIV-infected patients. J Clin Microbiol. 2001;39(12):4535-40

10. Phuong HT, Tran VC, Nguyen TV, Nguyen SH, Le DH, Truong TH, Nguyen $\mathrm{HL}$, Sugawara K, Tsuruta D. Case of Penicillium marneffei infection in a nonAIDS patient. J Dermatol. 2018;45(5):e104-5.

11. Yu X, Cai X, Xu X, Zhang L, Huang X, Wang L, Chen Y. Fungemia caused by Penicillium marneffei in an immunocompetent patient with COPD: a unique case report. Medicine. 2018:97(3):e9658.

12. Chan YF, Woo KC. Penicillium marneffei osteomyelitis. J Bone Joint Surgery British Volume. 1990;72(3):500-3.

13. Yap FB, Thevarajah $\mathrm{S}$, Asmah J. Penicillium marneffei infection in an African man. Dermatol Online J. 2010;16(7):2.

14. Duong TA. Infection due to Penicillium marneffei, an emerging pathogen: review of 155 reported cases. Clin Infect Diseases. 1996;23(1):125-30.

15. Tsang DN, Chan JK, Lau YT, Lim W, Tse CH, Chan NK. Penicillium marneffei infection: an underdiagnosed disease? Histopathology. 1988;13(3):311-8.

16. Wu TC, Chan JW, Ng CK, Tsang DN, Lee MP, Li PC. Clinical presentations and outcomes of Penicillium marneffei infections: a series from 1994 to 2004. Hong Kong Med J. 2008;14(2):103-9.

17. Vaidyanathan S, Patel CN, Scarsbrook AF, Chowdhury FU. FDG PET/CT in infection and inflammation--current and emerging clinical applications. Clin Radiol. 2015;70(7):787-800.

18. Chen D, Dong M, Zhao K, Sun F, Wang H, Liu Z. Unusual synchronous liver and brain abscesses infected by rare Aerococcus viridians in a patient with pulmonary arteriovenous malformations on FDG PET/CT: a case report and literature review. Medicine. 2017;96(49):e9048. 
19. Vos FJ, Bleeker-Rovers CP, Oyen WJ. The use of FDG-PET/CT in patients with febrile neutropenia. Semin Nucl Med. 2013;43(5):340-8.

20. Granados U, Fuster D, Pericas JM, Llopis JL, Ninot S, Quintana E, Almela M, Pare C, Tolosana JM, Falces C, et al. Diagnostic accuracy of 18F-FDG PET/CT in infective endocarditis and implantable cardiac electronic device infection: a cross-sectional study. J Nuclear Med. 2016;57(11):1726-32.

21. Rayamajhi SJ, Mittal BR, Maturu VN, Agarwal R, Bal A, Dey P, Shukla J, Gupta D. F-18-FDG and F-18-FLT PET/CT imaging in the characterization of mediastinal lymph nodes. Ann Nucl Med. 2016;30(3):207-16.

22. Hung CC, Hsueh PR, Chen MY, Hsiao CH, Chang SC, Luh KT. Invasive infection caused by Penicillium marneffei: an emerging pathogen in Taiwan. Clin Infectious Dis. 1998;26(1):202-3.

23. Jung JY, Jo GH, Kim HS, Park MY, Shin JH, Chin BS, Bang JH, Shin HS Disseminated penicilliosis in a Korean human immunodeficiency virus infected patient from Laos. J Korean Med Sci. 2012;27(6):697-700.

24. Ouyang Y, Cai S, Liang H, Cao C. Administration of Voriconazole in disseminated Talaromyces (Penicillium) Marneffei infection: a retrospective study. Mycopathologia. 2017;182(5):569-75.

\section{Publisher's Note}

Springer Nature remains neutral with regard to jurisdictional claims in published maps and institutional affiliations.

Ready to submit your research? Choose BMC and benefit from:

- fast, convenient online submission

- thorough peer review by experienced researchers in your field

- rapid publication on acceptance

- support for research data, including large and complex data types

- gold Open Access which fosters wider collaboration and increased citations

- maximum visibility for your research: over $100 \mathrm{M}$ website views per year

At BMC, research is always in progress.

Learn more biomedcentral.com/submissions 